\title{
Video Article \\ Laboratory Administration of Transcutaneous Auricular Vagus Nerve Stimulation (taVNS): Technique, Targeting, and Considerations
}

\author{
Bashar W. Badran ${ }^{* 1,2,3}$, Alfred B. Yu* ${ }^{2}$, Devin Adair ${ }^{1}$, Georgia Mappin ${ }^{3}$, William H. DeVries ${ }^{3}$, Dorothea D. Jenkins ${ }^{4}$, Mark S. George ${ }^{3,5,6}$, \\ Marom Bikson ${ }^{1}$ \\ ${ }^{1}$ Department of Biomedical Engineering, City College of New York \\ ${ }^{2}$ U.S. Army Research Laboratory, Aberdeen Proving Ground \\ ${ }^{3}$ Brain Stimulation Laboratory, Department of Psychiatry, Medical University of South Carolina \\ ${ }^{4}$ Department of Pediatrics, Medical University of South Carolina \\ ${ }^{5}$ Department of Neurology, Medical University of South Carolina \\ ${ }^{6}$ Ralph H. Johnson VA Medical Center \\ *These authors contributed equally
}

Correspondence to: Bashar W. Badran at basharwbadran@gmail.com

URL: https://www.jove.com/video/58984

DOI: doi:10.3791/58984

Keywords: Neuroscience, Issue 143, taVNS, tVNS, VNS, Transcutaneous Auricular Vagus Nerve Stimulation, vagus nerve stimulation, ear stimulation

\section{Date Published: 1/7/2019}

Citation: Badran, B.W., Yu, A.B., Adair, D., Mappin, G., DeVries, W.H., Jenkins, D.D., George, M.S., Bikson, M. Laboratory Administration of Transcutaneous Auricular Vagus Nerve Stimulation (taVNS): Technique, Targeting, and Considerations. J. Vis. Exp. (143), e58984, doi:10.3791/58984 (2019).

\section{Abstract}

Non-invasive vagus nerve stimulation (VNS) may be administered via a novel, emerging neuromodulatory technique known as transcutaneous auricular vagus nerve stimulation (taVNS). Unlike cervically-implanted VNS, taVNS is an inexpensive and non-surgical method used to modulate the vagus system. taVNS is appealing as it allows for rapid translation of basic VNS research and serves as a safe, inexpensive, and portable neurostimulation system for the future treatment of central and peripheral disease. The background and rationale for taVNS is described, along with electrical and parametric considerations, proper ear targeting and attachment of stimulation electrodes, individual dosing via determination of perception threshold (PT), and safe administration of taVNS.

\section{Video Link}

The video component of this article can be found at https://www.jove.com/video/58984/

\section{Introduction}

Cranial nerve $\mathrm{X}$, better known as the vagus nerve, is a large nerve tract that originates in the brainstem of the central nervous system and travels throughout the periphery, targeting every major organ in the thorax and abdomen (Figure 1) ${ }^{1}$. Vagus nerve stimulation (VNS) involves surgical implantation of bipolar electrodes around the left cervical branch of the vagus nerve. Electrical pulses are delivered to the vagus nerve via an implanted pulse generator (IPG) surgically implanted in the chest ${ }^{2}$. Although VNS is currently FDA-approved for epilepsy, refractory depression, and chronic obesity, it is a costly procedure requiring a hospital visit and surgery. Long-term safety of VNS is well established, and the majority of safety considerations regard current intensity related side effects (hoarse voice, throat pain) without serious stimulation-related adverse effects over the past 25 years of its clinical use ${ }^{3}$.

Recently, a noninvasive form of VNS known as transcutaneous auricular vagus nerve stimulation (taVNS) has emerged ${ }^{4}$. taVNS delivers electrical stimulation to the auricular branch of the vagus nerve (ABVN), an easily accessible target that innervates the human ear ${ }^{5}$. Over the last decade, several groups have demonstrated the safety and tolerability of this method ${ }^{6,7}$, including central and peripheral nervous system effects $^{9,10}$, and behavioral effects ${ }^{7,11,12,13}$ in neuropsychiatric populations. taVNS is also being explored in individuals as a promising enhancer of cognitive ${ }^{14,15}$ and social functioning ${ }^{16,17,18}$. As taVNS is becoming established, it offers the ability for researchers and clinicians to rapidly translate the promising VNS research that has been described in various disorders ranging from neurological and psychological trauma ${ }^{19,20,21}$, addiction $^{22}$, inflammation ${ }^{23}$, and tinnitus ${ }^{24,25}$.

In principle, taVNS is methodologically similar to conventionally administered transcutaneous electrical nerve stimulation (TENS) used to treat musculoskeletal pain disorders ${ }^{26}$. The difference is that taVNS is delivered to specific anatomical ear targets that are believed to be innervated by the $A B V N^{5}$. The field is still determining optimal stimulation targets ${ }^{27}$, although the two most common placements are the anterior wall of the outer ear canal (tragus) and the cymba conchae. Sham stimulation may be conducted by stimulating the earlobe of the ear, an area believed to have minimal ABVN innervation (Figure 2). Alternatively, sham may be delivered via a passive control method in which electrodes are attached to active sites, but no stimulation is delivered. Stimulation parameters may vary between groups, however according to the literature, stimulation 
is delivered in a pulsatile fashion (pulse width: $250-500 \mu \mathrm{s}$, frequency: $10-25 \mathrm{~Hz}$ ) and delivered at an individualized constant current ( $<5 \mathrm{~mA}$ ). Stimulation current varies by individual and experimental protocol, with many groups exploring various intensities as a function of an individual perceptual threshold (PT). The PT is defined as the minimum amount of current eliciting a perceived sensation at the target site and is usually determined via parametric estimation by customized sequential testing (PEST) software described in this report.

taVNS is a safe technique that may be administered in the laboratory or clinical setting. Side-effects of taVNS are minimal, with skin irritation or redness being the most common side-effect. Most taVNS studies explore stimulation of the left ear, as it is believed to be safer, although data in a large trial (Badran et al. 2018) reveal that right-sided stimulation has no increases in the risk of adverse events. Due to the wealth of literature in unilateral-left side stimulation, we will illustrate the typical taVNS set-up for laboratory studies investigating the use of left-sided taVNS as an intervention.

\section{Protocol}

This experimental protocol illustrates a typical taVNS set-up for use in a laboratory or clinical setting in which we target stimulating the anterior wall of the auditory canal (tragus) in a supine posture with an $8 \mathrm{~mm}$ diameter round metal electrode. These methods can be mimicked for alternative active treatment sites by simply changing electrode position to the cymba concha. All methods and procedures have been IRB approved by the Human Research Protection Program (HRPP) at City College New York.

\section{Materials}

1. Ensure all materials required to administer taVNS are prepared (Figure 3 ). The taVNS stimulator may be either a battery driven device that meets local safety regulations or powered from a conventional electrical outlet with built-in safety mechanisms that prevent unintended electrical surges. A constant current (current controlled) stimulator with a maximum output of $5 \mathrm{~mA}$ is required.

2. For taVNS, use stimulation electrodes made of a round conductive metal (tin, $\mathrm{Ag} / \mathrm{AgCl}$, gold) combined with a conductive medium such as electrolyte gel or conductive paste (see table of materials). Alternatively, use conductive electrodes made with flexible conductive carbon electrodes and conductive gel that may or may not be adhesive. Never place electrodes directly on the skin without a conductive medium, as this may pose unnecessary risk to the participant and can cause discomfort or pain.

3. Use computer running script software (see Table of Materials) that is programmed and used to control the stimulator and initiate stimulation with specific parameters. These parameters include current intensity $(\mathrm{mA})$, pulse width $(\mu \mathrm{s})$, frequency $(\mathrm{Hz})$, duty cycle $(\mathrm{On} / \mathrm{Off}$ time, s), session duration ( $\mathrm{min})$.

4. Use alcohol preparation pads ( $70 \%$ isopropyl alcohol) to prepare the skin surface before attaching electrodes to the ear. This removes surface oils from the skin surface and reduces the resistance of the skin, ensuring stimulation is delivered at safe power levels.

\section{Ear Targeting and Skin Preparation}

1. Use the following general inclusion criteria for conducting taVNS in the research setting: Age 18-70, no facial or ear pain, no recent ear trauma, no metal implants including pacemakers, not pregnant.

2. In the experiments involving healthy participants in a laboratory setting, use the following exclusion criteria: personal or family history of seizure, mood, or cardiovascular disorders, dependence on alcohol or recent illicit drug use, on any pharmacological agents known to increase seizure risk.

3. Seat the participant on a comfortable bed or chair in a supine or other relaxed position with legs elevated and head supported.

4. Inspect the left ear of the participant. Ensure no jewelry is attached and all make-up and lotion are removed. Confirm there are no skin-related contraindications at the site of stimulation, including sun burn, cuts, lesions, open sores.

5. Find the stimulation target, landmarked by the anterior wall of the outer ear canal externally by finding the tragus. Stimulation will be delivered to the portion of the ear canal directly behind the tragus (Figure 4).

6. Use an alcohol prep pad to gently scrub the target site, both internally and externally, to decrease skin resistance and increase conductance.

\section{Electrode Preparation and Placement}

1. If using non-disposable electrodes, visually inspect electrodes to ensure clean, corrosion-free surface is exposed. Ensure that electrodes are disinfected to prevent spread of bacteria between subjects. This can be done using alcohol or sterilization wipes to scrub the electrodes. If using disposable electrodes, skip to step 3.2.

2. Spread a thin layer of conductive paste to the surface of the electrode evenly. This will distribute electricity to the stimulation site. For an 8 $\mathrm{mm}$ diameter round electrode, a pea-sized amount of paste is sufficient. Spread the paste using a narrow wooden applicator to form a thin layer $<1 \mathrm{~mm}$ of paste on both electrodes.

3. Connect electrode cables to the stimulation device while the device is turned off and verify the polarity of the electrodes (red/positive electrode: anode, black/negative electrode: cathode). This is an important detail as targeting is polarity specific - the anode (red/positive terminal) is the electrode placed inside the ear canal and targeting the anterior wall of the outer ear canal. The cathode (black/negative terminal) sits on the outside of the ear attached to the tragus. For sham stimulation, the anode is placed on the anterior side of the ear.

4. Clip the spring electrode onto the tragus with the anode making contact with the anterior wall of the outer ear canal and the cathode contacting the anterior part of the tragus.

NOTE: If conducting sham stimulation, clip the electrode onto the earlobe (active control). Alternatively, sham stimulation may be delivered by attaching stimulation clips to active site and delivering no electrical current (passive control).

5. As subjects will feel the pressure of the electrodes clipped to their ear, ensure this pressure is not uncomfortable or disruptive to regional blood flow as demonstrated by pale white skin at clip site or physical pain sensed by the subject. After this point, determine perceptual threshold (PT) which will be described in the next procedural step. 


\section{Determination of Perceptual Threshold (PT)}

NOTE: Perceptual threshold is a critical value used to determine the power of taVNS stimulation. This value is defined as the minimum amount of electricity required to perceive electrical stimulation on the skin described as a pricking or tingling sensation.

1. Determine the PT using a simple step-up and step-down binary parametric search. First turn on the stimulator and set the output to 3 $\mathrm{mA}$. Deliver a 1 second train of taVNS stimulation at desired pulse width (typically $250-500 \mu \mathrm{s})$ and frequency $(25 \mathrm{~Hz}$, can vary based on application)

2. Ask the subject whether they felt the stimulation. Sensation is generally reported as a "tickle" or "pricking" sensation.

1. If YES, turn down stimulation intensity by $50 \%$ and repeat step 4.2 . If $\mathrm{NO}$, increase stimulation intensity by $50 \%$ and repeat step 4.2 .

3. Repeat the process described in step 4.2 until recording a minimum of 4 "YES" responses in which the $4^{\text {th }}$ YES response must come after a NO. The intensity (in $\mathrm{mA}$ ) of the PT will the value at which the subject says their fourth YES response to.

4. Use the example PT threshold finding is listed in Table 1 to assist in PT determination.

\section{Delivering Stimulation}

1. Once the subject is comfortable stimulation electrodes with properly attached to the desired target, and the perceptual threshold determined at the desired pulse width and frequency, begin the stimulation.

2. Use a computer running a pulse generating GUI (e.g., stimDesigner, freeware included with this manuscript) connected to a data acquisition unit (DAQ) to drive the stimulation system. The software should output TTL pulses as programmable settings (Figure 5). The TTL pulses will be sent via a BNC cable to the stimulator "trigger in" port. This interface software/stimulator interface allows modulation of frequency, duty cycle (on/off time), and session duration (Figure 6). The GUI used is attached as a free, open-source resource with this manuscript.

1. Ensure that stimulation is delivered at super-threshold levels, such as $200 \%$ of $P T^{8,9}$. For example, if the PT was determined to be 0.8 $\mathrm{mA}$, stimulation will be delivered at $1.6 \mathrm{~mA}$.

2. Ensure that the guidelines for duty cycles are followed when conducting long stimulation sessions. Typical duty cycles have $30-60 \mathrm{~s}$ "on" periods and $60-120 \mathrm{~s}$ "off" periods, or $20-50 \%$ duty cycles.

3. Vary the length of stimulation session (total time). Studies suggest that $30-60$ min stimulation sessions at a $25 \%$ duty cycle is safe and free of any acute side effects or adverse events. These sessions can be repeated with $12-24 \mathrm{~h}$ between sessions safely. NOTE: taVNS safety is unclear for longer periods of stimulation sessions, larger percentage duty cycles $(>40 \%)$, accelerated paradigms, and higher stimulation current doses.

\section{After taVNS}

1. When stimulation is completed, record objective data regarding the stimulation discomfort and side effects. Although taVNS, like implantable VNS, has limited safety concerns ${ }^{8,28}$, monitor and record sensation, discomfort, and any adverse events on a rating from $0-10^{29}$.

2. Remove the stimulation electrode from the ear and clean residual conductive paste from the subject's ear using an alcohol prep pad.

3. Use alcohol to clean and disinfect the stimulation electrode immediately upon removing from the subject's ear.

4. Inspect the ear for redness or irritation at the stimulation site and record any observations.

\section{Representative Results}

When proper skin preparation is conducted, perceptual thresholds are inversely correlated with stimulation pulse width. As pulse width increases the perceptual threshold decreases (Figure 7). Initial studies by this group exploring the effect of pulse width on PT in healthy individuals (meeting inclusion/exclusion criteria listed above), determined that the combined overall $(n=15,7$ female, mean age $26.5 \pm 4.99)$ PT at $100 \mu$ s $=$ $3.92 \pm 1.1 \mathrm{~mA} ; 200 \mu \mathrm{s}=2.24 \pm 0.74 \mathrm{~mA} ; 500 \mu \mathrm{s}=1.24 \pm 0.41 \mathrm{~mA}$. These thresholds suggest that a constant current stimulator with capacity of delivering up to $5 \mathrm{~mA}$ of current is required for stimulation of $500 \mu \mathrm{s}$ pulse width parameters, and a minimum of a $10 \mathrm{~mA}$ stimulator is required for lower pulse widths (Table 2). Fine tuning of the current is required, with increments of $0.1 \mathrm{~mA}$ are necessary for precise stimulation.

Delivering stimulation at $200 \%$ PT is tolerable and relatively pain free as demonstrated by pain numerical rating scales (NRS) scales ${ }^{9,30}$. The NRS scale is a rating system for pain from 0-10 in which individuals report pain or discomfort ${ }^{29}$. Both Active and Sham stimulation rate similarly low pain levels (NRS $<3$ for all stimulation pulse widths. More specifically, the biologically-active pulse width of $500 \mu \mathrm{s}$ delivered at $25 \mathrm{~Hz}$ is reported on average to rate as Active $=1.98 \pm 0.83$, Sham $=2.17 \pm 1.27(n=25,9$ female, mean age $25.16 \pm 4.16$ years $)$ (Table 3). Pain ratings for other parameters are no more painful than the $25 \mathrm{~Hz}$ parameter and the details can be found in the groups' prior work ${ }^{30}$.

Safety and tolerability of $30 \mathrm{~min}$ to 1 hour sessions at a $20-50 \%$ duty cycle has been widely reported in the literature with some studies delivering multiple sessions in the same day spread $12-15 \mathrm{~h}$ apart ${ }^{12,31}$. No serious adverse events have been reported from 60 subjects participating in several series of experiments with subjects participating from 1 to 8 repeated visits spread a minimum of 24 hours apart.

taVNS, when administered as reported in this manuscript, has been demonstrated to modulate the autonomic nervous system, induce functional brain activity changes as measured by fMRI BOLD, and piloted to treat neuropsychiatric disorders and aid in rehabilitation. 
A Circulation of Blood (oxygen)
Blood Pressure Regulation

Respiration Protein Production
Immune Response Toxin Clearance Absorption
Excretion Blood Filtration Immune Response Immune Response Digestion
Metabolism Appetite

Appetite Stomach /
Satiety Absorption Intestines Elimination

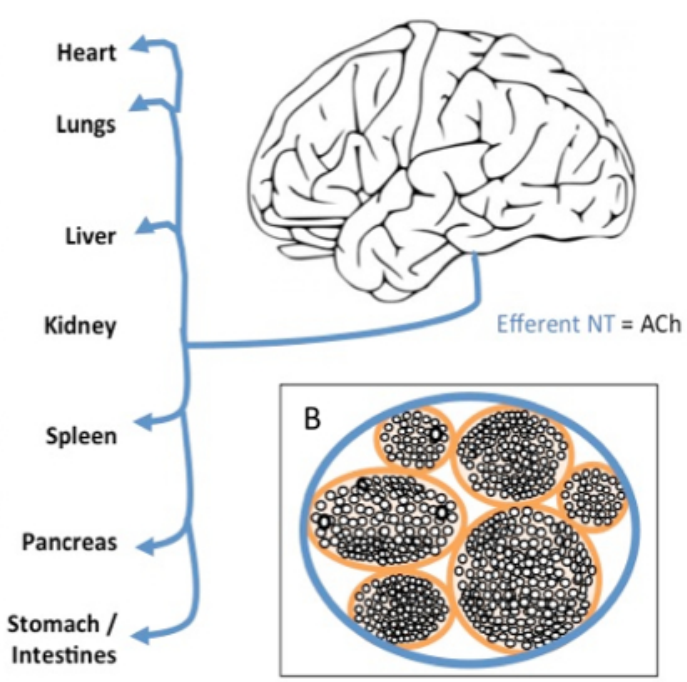

Figure 1: Vagus Nerve Efferent Projections and Cross-section. (A) Efferent projections of the vagus nerve target every major organ on the body with wide effects on bodily function (B) Cross-section of the vagus nerve, demonstrating the inside anatomy of the nerve as a series of bundles of nerves all contained within one major pathway. Please click here to view a larger version of this figure.

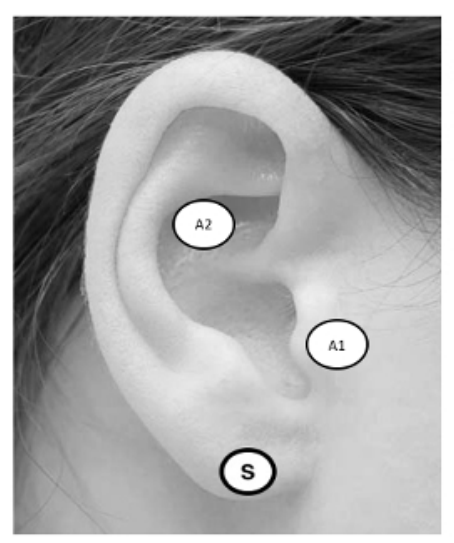

Figure 2: taVNS Ear Targets. Targeting the ABVN can be accomplished by stimulating the anterior wall of the outer ear canal, landmarked notably by the tragus (A1), or cymba conchae (A2). Sham stimulation is administered to the earlobe (S). Please click here to view a larger version of this figure.
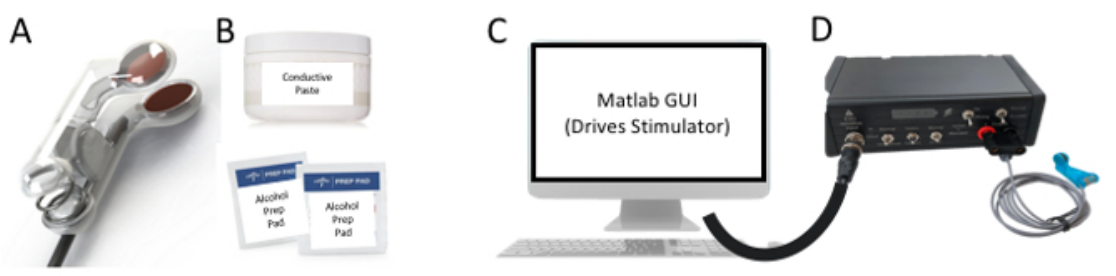

Figure 3: Key Components. The minimum required components for proper administration of taVNS are the following (A) ear stimulation electrodes, (B) conductive gel and alcohol prep pads, (C) computer capable of sending and receiving TTL pulses to a (D) constant current stimulator to trigger stimulation. Please click here to view a larger version of this figure. 


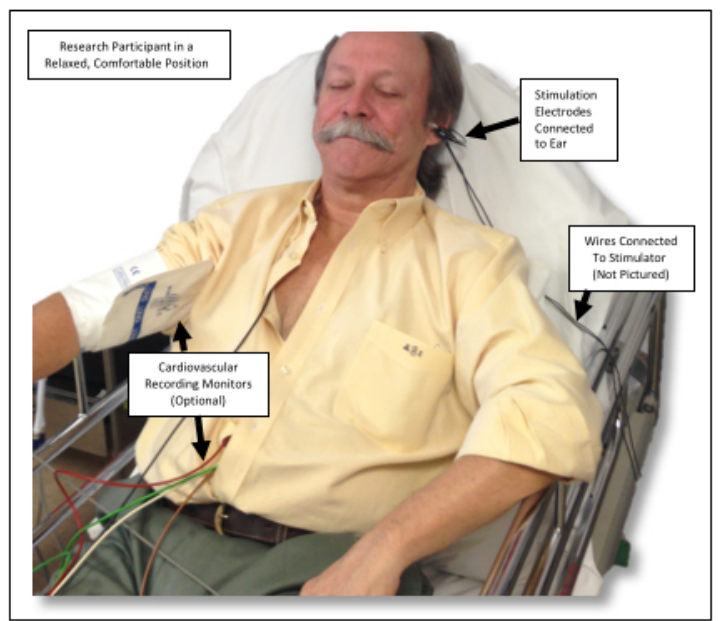

Figure 4: Example Setup. This photo shows an individual receiving taVNS of the left ear while in position to undergo an experimental paradigm. Please click here to view a larger version of this figure.

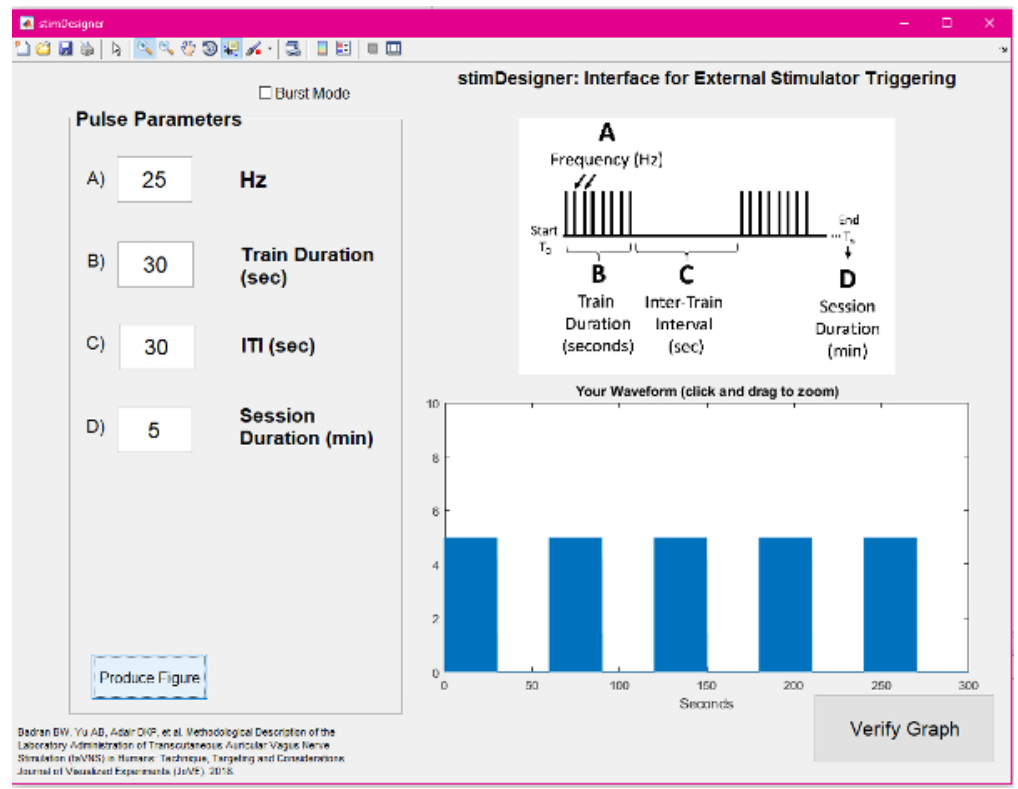

Figure 5: Screenshot of the GUI used for stimulation. Please click here to view a larger version of this figure. 


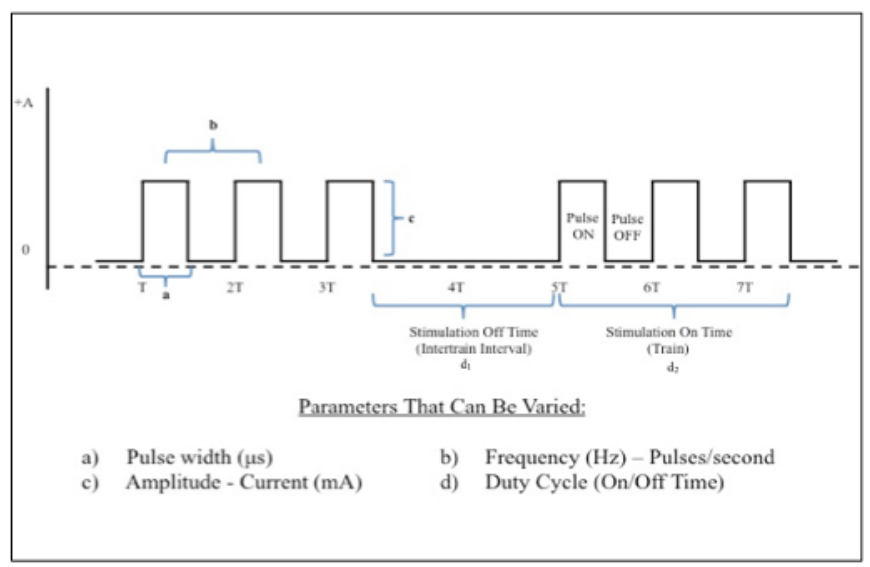

Figure 6: Electrical Stimulation Waveform Manipulations. Direct square wave electrical current can be delivered at various parameters. This figure demonstrates key properties of the waveform that can be changed in order to achieve desired biologic effects. Please click here to view a larger version of this figure.

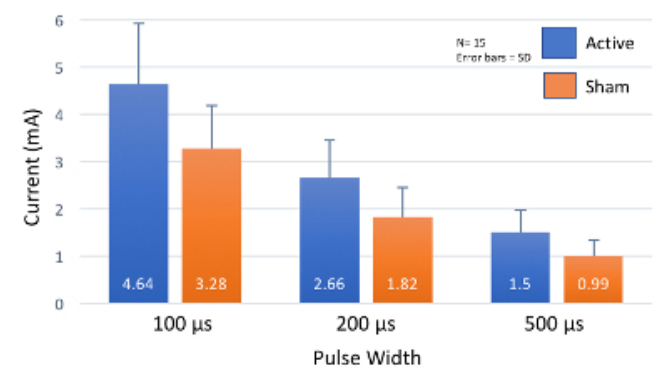

Figure 7: Perceptual Threshold Values at Increasing Pulse Widths. As pulse width increases, perceptual threshold (PT) decreases. Most healthy individuals will have a PT within 2 standard deviations (SD) of these mean values. Please click here to view a larger version of this figure.

\begin{tabular}{|c|c|c|c|c|c|c|}
\hline $\begin{array}{c}\text { Stimulation } \\
\text { Current } \\
\text { Setting }\end{array}$ & $3 \mathrm{~mA}$ & $1.5 \mathrm{~mA}$ & $0.75 \mathrm{~mA}$ & $1.1 \mathrm{~mA}$ & $0.55 \mathrm{~mA}$ & $0.825 \mathrm{~mA}$ \\
\hline Response & Yes & Yes & No & Yes & No & Yes \\
\hline
\end{tabular}

Table 1: Example of How to Determine Perceptual Threshold (PT). This table shows an example sequence of Yes/No responses used to parametrically determine PT.

\begin{tabular}{|c|c|c|c|}
\hline & \multicolumn{2}{|c|}{ Stim. Current \pm SD $(\mathrm{mA})$} & \\
\hline Pulse Width & $\begin{array}{c}\text { Tragus } \\
\text { (Active) }\end{array}$ & $\begin{array}{c}\text { Earlobe } \\
\text { (Control) }\end{array}$ & Sig \\
\hline $\mathbf{1 0 0} \boldsymbol{\mu} \mathbf{s}$ & $9.28 \pm 2.56$ & $6.57 \pm 1.83$ & Yes \\
\hline $\mathbf{2 0 0} \boldsymbol{\mu} \mathbf{s}$ & $5.32 \pm 1.60$ & $3.64 \pm 1.26$ & Yes \\
\hline $\mathbf{5 0 0} \boldsymbol{\mu} \mathbf{s}$ & $3 \pm 0.93$ & $1.97 \pm 0.71$ & Yes \\
\hline
\end{tabular}

Table 2: Stimulation Current Levels. Values of stimulation current in mA (200\% PT) for each pulse width ( $n=15)$.

\begin{tabular}{|c|c|c|c|c|c|c|c|c|c|}
\cline { 2 - 9 } \multicolumn{1}{c|}{} & \multicolumn{2}{c|}{ PT \pm SD $(\mathrm{mA})$} & Sig & \multicolumn{2}{|c|}{ Stimularon Curreat \pm SD $(\mathrm{mA})$} & Sig & \multicolumn{2}{c|}{ Pain \pm SD } & Sig \\
\hline & Active & Control & & Active & Control & & Active & Control & \\
\hline $500 \mu \mathrm{\mu s} 25 \mathrm{~Hz}$ & $0.82 \pm 0.41$ & $0.99 \pm 0.39$ & No & $1.37 \pm 0.68$ & $1.83 \pm 0.73$ & Yes & $1.98 \pm 0.83$ & $2.17 \pm 1.27$ & No \\
\hline
\end{tabular}

Table 3: PT, Stimulation Current, and Pain Values for Suggested Stimulation Parameters. Values of stimulation current in $\mathrm{mA}$ (200\% PT) for each pulse width $(n=25)$. 


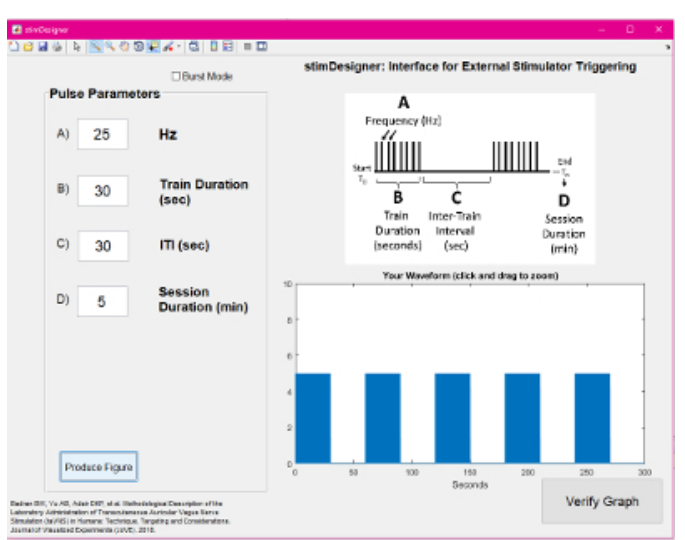

Supplemental File: Freeware GUI used in this protocol. Please click here to download this file.

\section{Discussion}

As in all novel modalities, all the described steps are critical in the safe administration of taVNS. Of ultimate concern is subject safety, which includes not only mitigating risks before taVNS via proper screening, but also monitoring subjects during stimulation for discomfort, pain, or adverse events. Here are the three most important consideration for administering taVNS. Screening for taVNS contraindications contraindications are as follows: any current or past history of cardiovascular disorders, facial or ear pain, recent ear trauma, metal implants above the level of the neck. For proper subject skin preparation, removing any surface oils, dirt, or makeup from the surface of the skin with alcohol helps with conductivity of the electrodes, reduces stimulation voltage required to drive the stimulator, and ultimately results in a more tolerable and safe stimulation session. It is encouraged to use a stimulator and electrodes meeting Low Output Transcranial Electrical Stimulation (LOTES) guidelines ${ }^{32}$. LOTES sets guidelines and industry standards for electrical stimulators that are built for stimulation of the head and neck and it is encouraged for groups to read this document before building their own systems. It is recommended to use either a FDAcleared plug-in stimulator (see Table of Materials), or a low voltage $(<50 \mathrm{~V})$, battery powered, constant current stimulator with appropriate safety measures built-in to avoid unintended over-delivery of current to the stimulation site. Ensure that electrodes are manufactured and assembled for specific use in taVNS. Ensure that the current manufacturing and engineering guidelines are followed as a reference if lab-made customized systems are used.

One consideration for taVNS is to ensure that the voltage output of the constant current stimulator can surmount the resistance of the skin and deliver the current required for stimulation. Ohm's law $(V=I R)$ demonstrates the relationship between current (I) and skin resistance (R). A minimum of a $20 \mathrm{~V}$ tabletop stimulator is recommended to avoid an underpowered system. Heat generated from the scalp or environment may degrade the conductive paste. If this occurs, it is recommended to stop stimulation and re-prep skin and electrodes with new conductive paste.

A limitation of taVNS is the vast parameter space. It is unknown as to which is more important - pulse width or frequency. There is a lacking data in recent taVNS trials that answer such questions. The various behavioral effects are derived from a variety of pulse widths, frequencies and stimulation currents $13,33,34,35,36,37,38,39$.

At this time, it is suggested that the $500 \mu$ s pulse width to be the most biologically active ${ }^{9}$. With respect to frequency, it has been demonstrated that $25 \mathrm{~Hz}$ is an effective frequency, although current investigations into optimal such as higher frequencies (>25 Hz), bilateral stimulation (left and right ears), and investigational burst paradigms are being conducted. Studies exploring different parameters of stimulation, alternative stimulation sites, and duty cycle optimization are needed to advance and refine the taVNS method.

taVNS is a promising non-invasive alternative to conventional VNS. taVNS provides an inexpensive $(<\$ 5,000$ in the demonstrated experimental setup, cost heavily dependent on type of stimulator used) and straightforward method that can be used to translate positive findings in animal models exploring the use of VNS on a variety of disorders, noninvasively modulate the autonomic nervous system, and potentially miniaturized and optimized for at-home neuromodulation for the treatment of neuropsychiatric and other disorders.

The future potential and possible applications of taVNS are vast. taVNS can serve as a promising adjunct or standalone treatment for neuropsychiatric disorders such as depression and epilepsy, taVNS-paired rehabilitation training to restore or accelerate learning of a behavior ${ }^{40}$, decrease inflammatory response ${ }^{41,42}$, and can potentially be used to enhance performance and autonomic function ${ }^{8,10}$.

\section{Disclosures}

The authors have nothing to disclose.

\section{Acknowledgements}

Research reported in this publication was supported by funding from the National Institutes of Health National Center of Neuromodulation for Rehabilitation, NIH/NICHD Grant Number P2CHD086844 which was awarded to the Medical University of South Carolina. The contents are solely the responsibility of the authors and do not necessarily represent the official views of the NIH or NICHD. 


\section{References}

1. Berthoud, H.R., \& Neuhuber, W.L. Functional and chemical anatomy of the afferent vagal system. Autonomic Neuroscience. 85 (1-3), 1-17 (2000).

2. George, M.S. et al. Vagus nerve stimulation: a new form of therapeutic Brain Stimulation. CNS Spectrums. 5 (11), 43-52 (2000).

3. Nemeroff, C.B. et al. VNS therapy in treatment-resistant depression: clinical evidence and putative neurobiological mechanisms. Neuropsychopharmacology. 31 (7), 1345-1355 (2006).

4. Ventureyra, E.C. Transcutaneous vagus nerve stimulation for partial onset seizure therapy. Child's Nervous System. 16 (2), $101-102$ (2000).

5. Peuker, E.T., \& Filler, T.J. The nerve supply of the human auricle. Clinical Anatomy. 15 (1), 35-37 (2002).

6. Kreuzer, P.M. et al. Transcutaneous vagus nerve stimulation: retrospective assessment of cardiac safety in a pilot study. Frontiers in Psychiatry. 3, 70 (2012).

7. Kreuzer, P.M. et al. Feasibility, safety and efficacy of transcutaneous vagus nerve stimulation in chronic tinnitus: an open pilot study. Brain Stimulation. 7 (5), 740-747 (2014).

8. Badran, B.W. et al. Short trains of transcutaneous auricular vagus nerve stimulation (taVNS) have parameter-specific effects on heart rate. Brain Stimulation. 11 (4), 699-708 (2018).

9. Badran, B.W. et al. Neurophysiologic effects of transcutaneous auricular vagus nerve stimulation (taVNS) via electrical stimulation of the tragus: A concurrent taVNS/fMRI study and review. Brain Stimulation. 11 (3), 492-500 (2018).

10. Clancy, J.A. et al. Non-invasive vagus nerve stimulation in healthy humans reduces sympathetic nerve activity. Brain Stimulation. 7 (6), 871-877 (2014).

11. Usichenko, T., Hacker, H., \& Lotze, M. Transcutaneous auricular vagal nerve stimulation (taVNS) might be a mechanism behind the analgesic effects of auricular acupuncture. Brain Stimulation. 10 (6), 1042-1044 (2017).

12. Rong, P. et al. Effect of transcutaneous auricular vagus nerve stimulation on major depressive disorder: A nonrandomized controlled pilot study. Journal of Affective Disorders. 195, 172-179 (2016).

13. Bauer, S. et al. Transcutaneous vagus nerve stimulation (tVNS) for treatment of drug-resistant epilepsy: a randomized, double-blind clinical trial (cMPsE02). Brain Stimulation. 9 (3), 356-363 (2016).

14. Jacobs, H.I., Riphagen, J.M., Razat, C.M., Wiese, S., \& Sack, A.T. Transcutaneous vagus nerve stimulation boosts associative memory in older individuals. Neurobiology of Aging. 36 (5), 1860-1867 (2015).

15. Jongkees, B.J., Immink, M.A., Finisguerra, A., \& Colzato, L.S. Transcutaneous Vagus Nerve Stimulation (tVNS) Enhances Response Selection During Sequential Action. Frontiers in Psychology. 9, 1159 (2018).

16. Sellaro, R., de Gelder, B., Finisguerra, A., \& Colzato, L.S. Transcutaneous vagus nerve stimulation (tVNS) enhances recognition of emotions in faces but not bodies. Cortex. 99, 213-223 (2018).

17. Jin, Y., \& Kong, J. Transcutaneous vagus nerve stimulation: a promising method for treatment of autism spectrum disorders. Frontiers in Neuroscience. 10 (2016).

18. Colzato, L.S., Ritter, S.M., \& Steenbergen, L. Transcutaneous vagus nerve stimulation (tVNS) enhances divergent thinking. Neuropsychologia. 111, 72-76 (2018).

19. George, M.S. et al. Vagus nerve stimulation for the treatment of depression and other neuropsychiatric disorders. Expert Review of Neurotherapeutics. 7 (1), 63-74 (2007).

20. Kong, J., Fang, J., Park, J., Li, S., \& Rong, P. Treating Depression with Transcutaneous Auricular Vagus Nerve Stimulation: State of the Art and Future Perspectives. Frontiers in Psychiatry. 9, 20 (2018).

21. Dawson, J. et al. Safety, feasibility, and efficacy of vagus nerve stimulation paired with upper-limb rehabilitation after ischemic stroke. Stroke. 47 (1), 143-150 (2016).

22. Liu, H. et al. Vagus nerve stimulation inhibits heroin-seeking behavior induced by heroin priming or heroin-associated cues in rats. Neuroscience Letters. 494 (1), 70-74 (2011).

23. Zhang, Y. et al. Chronic Vagus Nerve Stimulation Improves Autonomic Control and Attenuates Systemic Inflammation and Heart Failure Progression in a Canine High-Rate Pacing ModelCLINICAL PERSPECTIVE. Circulation: Heart Failure. 2 (6), $692-699$ (2009).

24. De Ridder, D., Kilgard, M., Engineer, N., \& Vanneste, S. Placebo-controlled vagus nerve stimulation paired with tones in a patient with refractory tinnitus: a case report. Otology \& Neurotology. 36 (4), 575-580 (2015).

25. Shim, H.J. et al. Feasibility and safety of transcutaneous vagus nerve stimulation paired with notched music therapy for the treatment of chronic tinnitus. Journal of Audiology \& Otology. 19 (3), 159-167 (2015).

26. Chesterton, L.S., Foster, N.E., Wright, C.C., Baxter, G.D., \& Barlas, P. Effects of TENS frequency, intensity and stimulation site parameter manipulation on pressure pain thresholds in healthy human subjects. Pain. 106 (1-2), 73-80 (2003).

27. Badran, B.W. et al. Tragus or cymba conchae? Investigating the anatomical foundation of transcutaneous auricular vagus nerve stimulation (taVNS). Brain Stimulation. 11 (4), 947-948 (2018).

28. Ramsay, R.E. et al. Vagus nerve stimulation for treatment of partial seizures: 2 . Safety, side effects, and tolerability. First International Vagus Nerve Stimulation Study Group. Epilepsia. 35 (3), 627-636 (1994).

29. Farrar, J.T., Young, J.P., Jr., LaMoreaux, L., Werth, J.L., \& Poole, R.M. Clinical importance of changes in chronic pain intensity measured on an 11-point numerical pain rating scale. Pain. 94 (2), 149-158 (2001).

30. Badran, B.W. et al. Short trains of transcutaneous auricular vagus nerve stimulation (taVNS) have parameter-specific effects on heart rate. Brain Stimulation. (2018).

31. Bauer, S. et al. Transcutaneous Vagus Nerve Stimulation (tVNS) for Treatment of Drug-Resistant Epilepsy: A Randomized, Double-Blind Clinical Trial (cMPsE02). Brain Stimulation. 9 (3), 356-363 (2016).

32. Bikson, M. et al. Limited output transcranial electrical stimulation (LOTES-2017): Engineering principles, regulatory statutes, and industry standards for wellness, over-the-counter, or prescription devices with low risk. Brain Stimulation. 11 (1), 134-157 (2018).

33. Kraus, T. et al. BOLD fMRI deactivation of limbic and temporal brain structures and mood enhancing effect by transcutaneous vagus nerve stimulation. Journal of Neural Transmission. 114 (11), 1485-1493 (2007).

34. Kraus, T. et al. CNS BOLD fMRI effects of sham-controlled transcutaneous electrical nerve stimulation in the left outer auditory canal-a pilot study. Brain Stimulation. 6 (5), 798-804 (2013). 
35. Fallgatter, A. et al. Far field potentials from the brain stem after transcutaneous vagus nerve stimulation. Journal of Neural Transmission. 110 (12), 1437-1443 (2003).

36. Fallgatter, A.J., Ehlis, A.-C., Ringel, T.M., \& Herrmann, M.J. Age effect on far field potentials from the brain stem after transcutaneous vagus nerve stimulation. International Journal of Psychophysiology. 56 (1), 37-43 (2005).

37. Polak, T. et al. Far field potentials from brain stem after transcutaneous vagus nerve stimulation: optimization of stimulation and recording parameters. Journal of Neural Transmission. 116 (10), 1237-1242 (2009).

38. Greif, R. et al. Transcutaneous electrical stimulation of an auricular acupuncture point decreases anesthetic requirement. The Journal of the American Society of Anesthesiologists. 96 (2), 306-312 (2002).

39. Wang, S.-M., Peloquin, C., \& Kain, Z.N. The use of auricular acupuncture to reduce preoperative anxiety. Anesthesia \& Analgesia. 93 (5), 1178-1180 (2001).

40. Badran, B.W. et al. Transcutaneous auricular vagus nerve stimulation (taVNS) for improving oromotor function in newborns. Brain Stimulation: Basic, Translational, and Clinical Research in Neuromodulation. (2018).

41. Borovikova, L.V. et al. Vagus nerve stimulation attenuates the systemic inflammatory response to endotoxin. Nature. 405 (6785), $458-462$ (2000).

42. Ulloa, L. The vagus nerve and the nicotinic anti-inflammatory pathway. Nature Reviews Drug Discovery. 4 (8), 673-684 (2005). 